\title{
Preparation, Characterization, and Biological Testing of Novel Magnetic nanocomposite hydrogels
}

Jianghong Huang ${ }^{1,2,}$, , Yujie Liang ${ }^{3}$, Zhiwang Huang ${ }^{1,2}$, Jianyi Xiong ${ }^{1,2}$, Daping Wang ${ }^{1,2, *}$

1. Shenzhen National Key Department of Orthopedics, Shenzhen Second People's Hospital (The First Hospital Affiliated to Shenzhen University), Shenzhen 518035, P. R China.

2. Shenzhen Key Laboratory of Tissue Engineering, Shenzhen Laboratory of Digital Orthopedic Engineering, Shenzhen Second People's Hospital (The First Hospital Affiliated to Shenzhen University), Shenzhen 518035, P. R China.

3. Shenzhen Kangning Hospital, Shenzhen Mental Health Center, Shenzhen, Guangdong Province, P. R China.

*Address correspondence: huangjianghong88@sohu.com, dapingwang1963@qq.com. 


\section{Supplemental Material}

Figure S1: A: Mechanical test of each group of hydrogel matrices. B: Mechanical testing of each group of new Magnetic nanocomposite hydrogels




Figure S2: A: Bone marrow mesenchymal stem cells P1. B: Bone marrow mesenchymal stem cells P3. C: Bone marrow mesenchymal stem cells were co-cultured with new Magnetic nanocomposite hydrogel for the third day. D: Bone marrow mesenchymal stem cells were co-cultured with a new Magnetic nanocomposite hydrogel for 7 days. It can be seen that the cells proliferate well on the surface of the material.
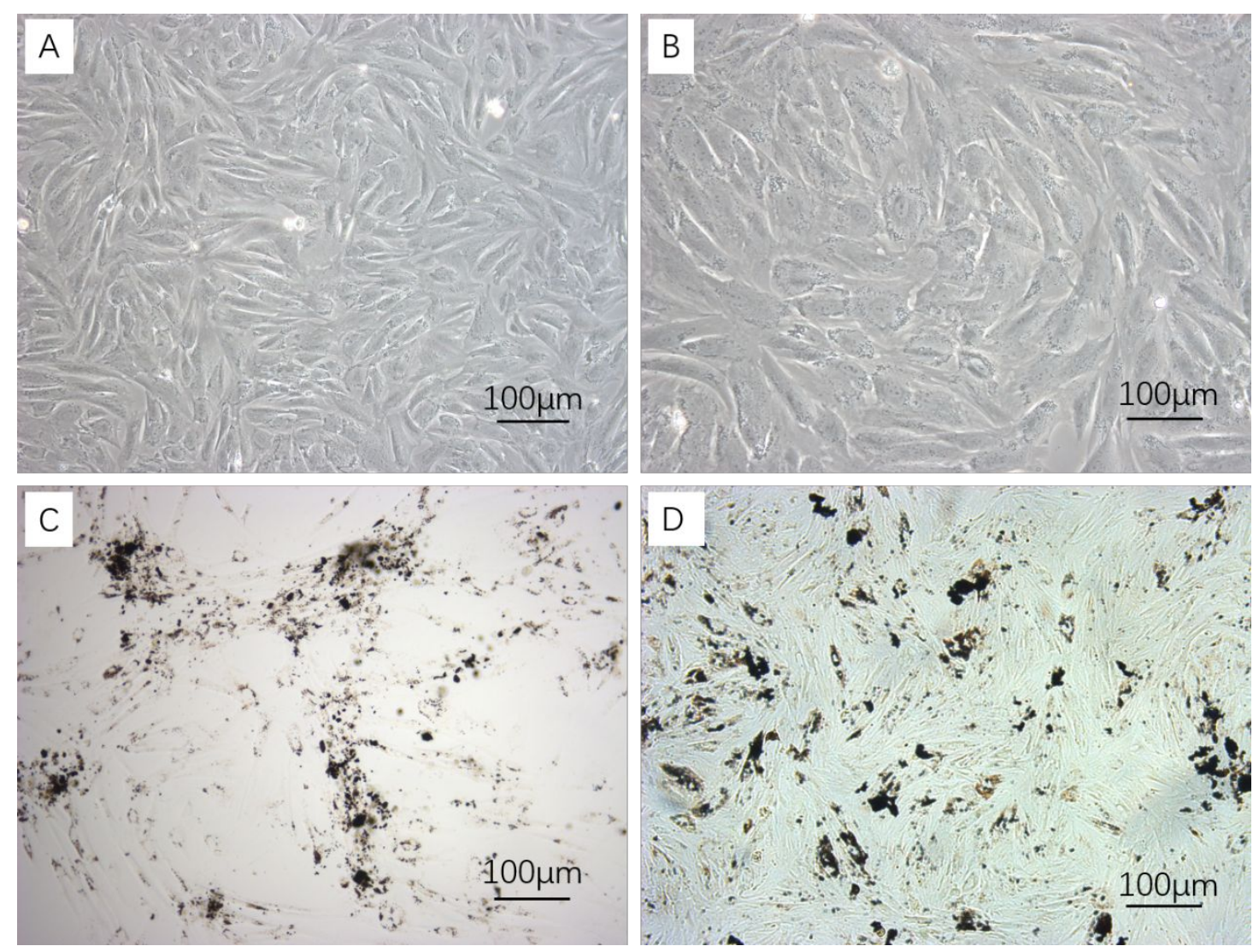
Figure S3: Magnetic field responsiveness test of the new Magnetic nanocomposite hydrogel. The test results show that the new Magnetic nanocomposite hydrogel has magnetic field responsiveness and can be attracted by magnets.

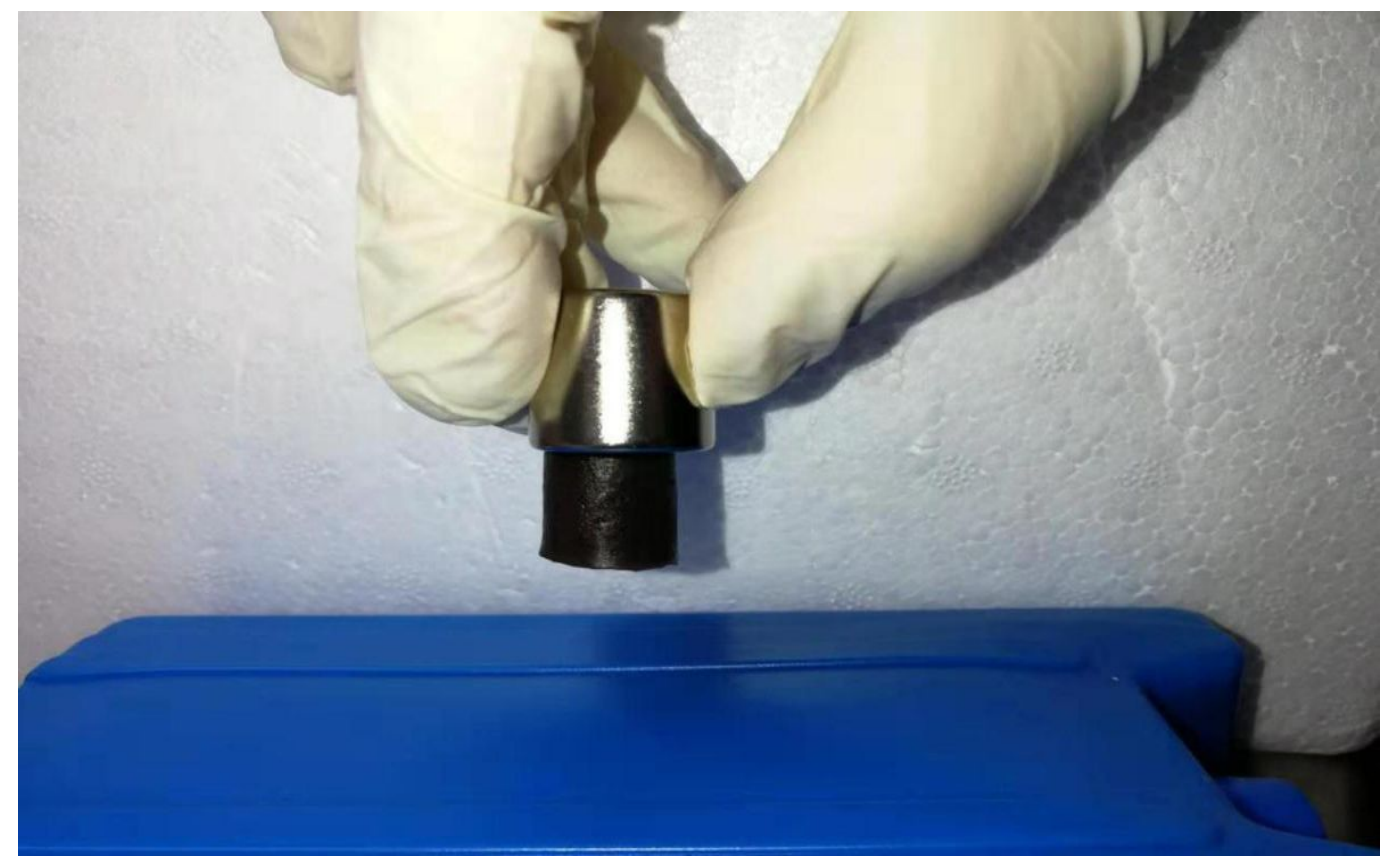

